\title{
émulations
}

\section{Enjeux et dynamiques de bureaucratisation des pratiques associatives en milieu rural}

\section{L'exemple de l'association bambourang-ba dans la commune d'Adéane (Sénégal)}

Koly Fall

Émulations - Revue de sciences sociales, 2021, $\mathrm{n}^{\circ} 37$, «Associations et bureaucratisation : perspectives africaines ».

\section{Article disponible à l'adresse suivante}

https://ojs.uclouvain.be/index.php/emulations/article/view/fall

\section{Pour citer cet article}

Koly Fall, « Enjeux et dynamiques de bureaucratisation des pratiques associatives en milieu rural. L'exemple de l'association bambourang-ba dans la commune d'Adéane (Sénégal) », Émulations, n³7, Mise en ligne le 19 mars 2021.

DOI : 10.14428/emulations.037.05

Distribution électronique : Université catholique de Louvain (Belgique) : ojs.uclouvain.be

(C) Cet article est mis à disposition selon les termes de la Licence Creative Commons Attribution, Pas d'Utilisation Commerciale 4.0 International. http://creativecommons.org/licenses/by-nc/4.0/

Éditeur : Émulations - Revue de sciences sociales / Presses universitaires de Louvain https://ojs.uclouvain.be/index.php/emulations

ISSN électronique : 1784-5734

PUL PRESSES

UNIVERSITAIRES

DE LOUVAIN 


\title{
Enjeux et dynamiques de bureaucratisation des pratiques associatives en milieu rural ${ }^{1}$
}

\author{
L'exemple de l'association bambourang-ba \\ dans la commune d'Adéane (Sénégal)
}

Koly Fall ${ }^{2}$

\begin{abstract}
[Résumé] Cet article est une analyse du processus de bureaucratisation des pratiques associatives en milieu rural. II propose une étude de cas de l'association bambourang-ba dans la commune d'Adéane du département de Ziguinchor (Sénégal). À première vue, bambourang-ba apparaît comme une structure autonome constituée d'acteurs locaux qui nouent un partenariat avec l'ONG ChildFund et la fédération dimbaya. Toutefois, les données révèlent une dynamique d'organisation et de gestion insufflée par le haut et qui répond à une exigence de contrôle de l'ONG et son partenaire local. Cette dynamique de bureaucratisation se complexifie au fur et à mesure que les activités de l'ONG (le parrainage) s'étendent dans le département de Ziguinchor. Elle est coachée par des agents de terrain qui mobilisent des ressources variées et encadrent son appropriation par les dirigeants de l'association. Enfin, l'article propose une analyse de l'intermédiation et des dynamiques de bureaucratisation autour du parrainage des enfants.
\end{abstract}

Mots clés : bureaucratisation, ChildFund, parrainage, association, pratiques bureaucratiques.

Stakes and dynamics of bureaucratization of associative practices in rural areas. The example of the bambourang-ba association in the commune of Adeane (Senegal)

[Abstract] This article is an analysis of the process of bureaucratization of associative practices in rural areas. It proposes a case study of the bambourang-ba association in the commune of Adeane in the department of Ziguinchor (Senegal). At first glance, bambourang-ba appears to be an autonomous structure made up of local actors who form a partnership with the NGO ChildFund and the dimbaya federation. However, the data reveal an organizational and management dynamic that is driven from the top and that meets a need for control by the NGO and its local partner. This dynamic of bureaucratization becomes more complex as the NGO's activities (sponsorship) expand into the department of Ziguinchor. It is coached by field agents who mobilize various resources and supervise its appropriation by the association's leaders. Finally, the article proposes an analysis of intermediation and the dynamics of bureaucratization around sponsorship.

Keywords: bureaucratization, ChildFund, sponsorship, association, bureaucratic practices.

\section{Introduction}

En Afrique, les études sur la bureaucratie ont longtemps été centrées sur le système et le mode de fonctionnement des administrations publiques. Elles ont permis de mettre

\footnotetext{
${ }^{1}$ Cet article a été réalisé grâce à l'appui financier de I'IHA-CREPOS.

2 Université Cheikh Anta Diop de Dakar, LASAP, IHA-CREPOS, Sénégal.
} 
en évidence les failles de ces dernières à travers l'histoire de L'État importé (Badie, 1992), La greffe de l'État (Bayart, 1996), la corruption (Blundo, Olivier de Sardan, 2007) et la mobilisation de normes pratiques (Olivier de Sardan, 2008). Dans le domaine associatif, la bureaucratie s'analyse sous l'angle des pratiques bureaucratiques, entendues comme des dispositions réglementaires (formelles ou formalisées) à caractère administratif (Weller, 2018). Celles-ci sont perceptibles à travers l'influence des projets de développement dans la gouvernance associative. Les organisations et organismes internationaux ont mis en place des architectures institutionnelles (Olivier de Sardan, 2009:25) et transmettent des savoir-faire (Niang, 2000) techniques en vue de dynamiser les initiatives de développement local.

En effet, les études consacrées aux associations et groupements ruraux en Afrique insistent généralement sur leur rôle " d'intermédiaires » par lesquels transite la rente du développement de l'État, mais aussi des projets et programmes internationaux (Bierschenk, Chauveau, Olivier de Sardan, 2000). Ils sont présentés comme des espaces où « la logique bureaucratique de l'État central se confronte et négocie avec d'autres logiques d'action et diverses représentations locales du politique » (Ibid.: 10). Par ailleurs, les analyses du fonctionnement de ces structures associatives mettent en évidence plusieurs blocages liés à la difficulté d'adaptation des modèles et procédures imposées par les projets (Behrends, Sung-Joon, Rottenburg, 2014 ; Olivier de Sardan, Diarra, Moha, 2017). Dans le prolongement de ces réflexions, cet article propose une analyse de la bureaucratisation de ces associations par les ONG (organisations non gouvernementales) en milieu rural dans la région de Ziguinchor. Il s'insère dans une problématique générale autour de l'enjeu de l'utilisation et de l'appropriation des pratiques bureaucratiques par ces associations dans leur dynamique de collaboration avec des ONG étrangères.

L'installation et l'intervention des ONG, au cours des dernières décennies, dans la région de Ziguinchor (au sud du Sénégal), sont fortement liées à l'insécurité et au conflit armé entre les combattants du MFDC (Mouvement des forces démocratiques de la Casamance) et l'armée du Sénégal. Ce conflit a entraîné une situation de « ni guerre ni paix entrecoupée par des attaques meurtrières » (Ébère, 2015 : 60), des ravages de villages et destructions de biens matériels, et des déplacements forcés de populations dont le retour pose aujourd'hui encore d'énormes difficultés (Robin, 2006). Le 15 juin 2020, l'explosion d'une mine a causé la mort de deux soldats de l'armée sénégalaise lors d'une opération de sécurisation du retour des réfugiés du village de Bissine, dans la commune d'Adéane. Ainsi, la fragmentation du MFDC en différentes factions et la multiplication des attaques dans les années 1990 ont conduit l’État du Sénégal à reconnaître ses limites dans la gestion de la question casamançaise (Foucher, 2007). L'implication d'acteurs de la société civile à la veille des années 2000 se présentait plus comme une exigence qu'une simple volonté des autorités politiques locales et nationales. C'est ainsi que plusieurs projets, ONG et organismes se sont installés dans la région. 
Dans leur dynamique d'intervention et pour faciliter la mise en œuvre de leurs projets, ces ONG et organismes ont structuré les destinataires de leurs programmes en associations dont la forme intègre les ressources et stratégies des acteurs locaux. Dans le département de Ziguinchor, l'ONG ChildFund a mis en place un réseau de six associations dans les communes de Boutoupa Camaracounda (Najoudapot) au sud-est, de Niassya (Amona) au sud-ouest, d'Enampore (Kaputen) au nord-ouest, de Niaguis (Dinding yirwando) au nord, de Ziguinchor (Dinding kafo) au centre et d'Adéane (Bambourang-ba) au nord-est. Pour faciliter l'encadrement de ce réseau, l'ONG a mis en place un dispositif organisationnel qui a conduit, en 2009, à la création de la fédération dimbaya kagnalen ${ }^{3}$ en regroupant les six associations. Ce dispositif mobilise des instruments et des « formalités bureaucratiques » variées (Hibou, 2013 : 9). Son analyse met en évidence une dynamique de bureaucratisation par le haut qui s'inscrit plus globalement dans la logique des ONG qui tentent de transférer un «modèle d'organisation bureaucratique pensé comme universel tant au plan de sa légitimité que de son efficacité »(Chauveau, 1994 : 42). Son intégration et son appropriation par les communautés sont une condition à l'intervention de l'ONG ChildFund dans le département de Ziguinchor. Elles sont encadrées (coachées) par les agents de terrain de ChildFund et de dimbaya, et révèlent une forme de « participation forcée » des communautés.

Cette étude porte sur le cas de l'association bambourang-ba $a^{4}$, dans la commune d'Adéane où nous menons une recherche doctorale sur la solidarité dans les organisations communautaires ${ }^{5}$. Elle adresse la question de recherche suivante : comment l'association bambourang-ba, en assurant le rôle d'intermédiaire entre l'ONG ChildFund et ses bénéficiaires, devient-elle un espace de bureaucratisation et d'appropriation locale des instruments et pratiques bureaucratiques ? Pour aborder cette problématique, nous analysons dans un premier temps l'implantation de ChildFund et la mise en place de son réseau associatif dans le département de Ziguinchor. Cela permettra d'appréhender l'intégration des pratiques bureaucratiques dans le fonctionnement du dispositif organisationnel mis en place par l'ONG, et les dynamiques d'ancrage de l'association bambourang-ba à Adéane. Dans un second temps, nous étudions le rôle des agents de terrain dans l'appropriation des pratiques bureaucratiques insufflées par l'ONG. Enfin, nous analysons l'enjeu et les dynamiques de bureaucratisation autour du parrainage des enfants à Adéane.

\footnotetext{
${ }^{3}$ Dimbaya kagnalen est le nom donné au rituel consacré aux femmes qui ont des difficultés pour avoir des enfants.

${ }^{4}$ Bambourang-ba est un terme mandingue qui désigne le pagne utilisé par la femme pour couvrir l'enfant porté sur le dos. Selon nos interlocuteurs, ce pagne protège l'enfant contre les mauvais sorts et les mauvais esprits.

${ }^{5}$ Je remercie mon directeur de thèse, Souleymane Gomis, mes collègues de l'IHA-CREPOS et Paul Diédhiou d'avoir commenté ce texte.
} 


\section{Démarche méthodologique}

Les données mobilisées dans cet article sont issues d'une série d'enquêtes réalisées entre octobre 2018 et janvier 2020 à Adéane et à Ziguinchor. Nous avons réalisé quinze entretiens semi-directifs autour des thèmes suivants : le parcours et l'expérience des enquêtés ; l'historique et l'installation de l'ONG ChildFund dans le département de Ziguinchor ; la gestion de l'association bambourang-ba, son fonctionnement et ses activités à Adéane ; l'organisation du parrainage des enfants ; les outils de gestion de l'association et les contraintes liées à leur utilisation ; etc. Nous avons interrogé les dirigeants de l'association bambourang-ba (le président, la trésorière, etc.) à Adéane et le responsable du staff technique et du parrainage à la fédération dimbaya à Ziguinchor. Le président de l'association a demandé à faire l'entretien en présence du mobilisateur communautaire qu'il considère comme son «supérieur et le représentant de l'ONG et de la fédération ». En outre, nous avons interrogé trois relais, quatre mobilisateurs communautaires et le maire de la commune d'Adéane, entre autres.

Le choix des personnes enquêtées a été généralement fondé sur leur expérience et leur implication dans la gestion de l'association et la mise en œuvre des activités et projets de l'ONG et de la fédération dans la commune d'Adéane. Nous avons utilisé la technique de boule de neige qui a consisté à demander aux " premiers enquêtés d'indiquer d'autres personnes qui sont concernées par l'étude» (Combessie, 2007 : 53). Les entretiens ont été réalisés en français (avec les agents de terrain) et en wolof (avec certains dirigeants de l'association). Ils ont été combinés avec l'observation de trois réunions de travail du bureau de l'association et du staff technique de la fédération qui est chargé du suivi des activités de terrain. Par ailleurs, l'analyse du processus de bureaucratisation et d'appropriation des pratiques bureaucratiques conduit à s'intéresser au rôle des instruments (Lascoumes, Le Galès, 2005) : règlement intérieur, rapports d'activités, justificatifs de dépenses, entre autres. C'est pourquoi nous avons eu recours à l'analyse documentaire en exploitant les textes règlementaires et les outils de gestion de l'association.

Enfin, lors des entretiens, le responsable du staff technique et le mobilisateur communautaire ont expliqué que l'accès à certains documents était restreint pour des raisons de confidentialité. Il s'agit des documents de création de l'association et de ses démembrements, mais aussi des plans d'action sur les principales orientations de l'association et des dispositions mises en place par l'ONG pour leur réalisation :

Si des personnes extérieures à l'ONG ou de la fédération ont accès à ces documents, elles peuvent facilement reproduire notre modèle ailleurs sans qu'on ait le pouvoir de les en empêcher. C'est pourquoi, pour chaque document, il y a un code d'accès que vous ne pouvez pas obtenir si vous n'avez pas une habilitation de l'ONG. Donc, même si je voulais vous les donner, je ne pourrais pas, car je n'y ai pas accès. (M.T ${ }^{6}$ mobilisateur communautaire)

\footnotetext{
${ }^{6}$ Nous avons adopté ce mode de citation codé qui fournit peu d'information sur les profils de manière à garantir l'anonymat des enquêtés.
} 
Pour contourner ce blocage, nous avons eu recours aux entretiens individuels ; ce qui nous a permis de recueillir des données sur la formation de l'association et ses démembrements, notamment les associations villageoises d'épargne et de crédit (AVEC).

\section{1. Évolution et dynamique du mouvement associatif à Ziguinchor}

Parmi les premières associations urbaines dans la région de Ziguinchor, nous pouvons citer les Usana (fromager en diola). Les Usana ont émergé dans la ville de Ziguinchor entre 1930 et 1940 (Foucher, 2007). Constituées de femmes et très impliquées dans la gestion des affaires locales, ces associations rituelles ont recours aux valeurs autour des cérémonies de prière et de propitiation (Ibid. : 62). Après la Seconde Guerre mondiale, le mouvement associatif de la région a connu un essor considérable. Les associations confrériques religieuses se sont multipliées en milieu urbain ziguinchorois avec la formation du « Groupement Qâdriya » autour de l'entraide et la défense des intérêts des disciples (Trincaz, 1984 : 221). Cette dynamique s'est renforcée au lendemain des indépendances avec la création de plusieurs associations et clubs de jeunes et d'adultes jusque dans les années 1970.

À côté des mouvements de jeunesse (les Éclaireurs, les scouts, la JEC - Jeunesse estudiantine catholique, etc.) et des associations sportives et culturelles, plusieurs associations de ressortissants se sont formées dans les centres urbains, à Ziguinchor et à Bignona. Souvent constituées de façon spontanée, ces associations sont majoritairement composées de migrants travailleurs ou à la recherche d'un emploi et appartenant à la même communauté villageoise, ethnique ou religieuse (Dupuy, 1990). Elles permettent à leurs membres de développer une solidarité centrée autour de "l'adaptation en milieu urbain, de l'affirmation de l'appartenance à un groupe social fondée sur la tradition » (Trincaz, 1984 : 218), et du « développement d'activités socioéconomiques et récréatives » (Awenengo, 2006:5).

Toutefois, au cours de la décennie 1980, les associations ont suscité moins d'intérêt scientifique pour les spécialistes de la Casamance7. Pour sa part, la décennie 1990 a été entamée par des négociations entre les responsables du MFDC et le gouvernement du Sénégal avec la signature d'un accord de paix en 1991 (Foucher, 2007). Cependant, le conflit s'est complexifié, d'une part, avec la multiplication des affrontements et attaques à main armée ayant abouti à plusieurs centaines de déplacés. Jusqu'en 2013, les estimations indiquaient entre « 24000 et 40000 déplacés internes et des dizaines de milliers de réfugiés dans les pays voisins » (Ébère, 2015 : 60). D’autre part, la fragmentation du MFDC en différentes factions (Foucher, 2007 ; Robin, 2006) a diversifié les interlocuteurs de l'État et rendu plus difficile une solution de paix durable.

Cette situation aurait ouvert un " espace de négociation et de compétition entre différents acteurs et communautés en quête de reconnaissance et de visibilité qui dépasse

\footnotetext{
${ }^{7}$ Cela s'est traduit par une quasi-absence d'études portant sur le mouvement associatif de la région durant la période 1980-1990. Cette remarque faite lors de mes recherches bibliographiques conduit à s'interroger sur les conséquences du conflit sur le choix des problématiques de recherche en Casamance, particulièrement à Ziguinchor.
} 
la simple question de leur intégration dans le processus de paix » (Awenengo, 2006 : 10). Cela s'est traduit par une forte mobilisation des femmes et la prise en compte de la dimension culturelle dans le processus de paix (Foucher, 2007). L'une des premières initiatives dans ce domaine a été la création de l'Association culturelle Aguène et Diambogne en 1994 par Saliou Sambou, alors gouverneur de la région de Ziguinchor (Awenengo, 2006). Par la suite, plusieurs autres associations culturelles ont vu le jour à Ziguinchor au nom de la paix et de la cohésion. Cette dynamique s'est intensifiée à partir de la seconde moitié de la décennie 1990. En libéralisant le marché de la paix en Casamance, l'État du Sénégal a incité et facilité l'implantation d'acteurs privés dans la région. Il s'en est suivi une floraison d'ONG locales (Dynamique de paix, Usoforal, Kabonketor, les Femmes du bois sacré, etc.) et d'organismes étrangers (USAID, COSPE, ChildFund, Handicap international, Futur au Présent, etc.).

Basées dans la ville de Ziguinchor, la plupart de ces organisations interviennent en milieu rural à travers des programmes d'aide et de soutien (financer des groupements de femmes, réhabiliter des habitations, construire des écoles, etc.) aux familles affectées par le conflit. Certaines ONG ont adopté une stratégie visant à regrouper leurs bénéficiaires en associations villageoises mobilisées pour la paix, l’intégration socioéconomique et la valorisation des actions des ONG et organismes partenaires. Au sein de ces structures associatives se confrontent différents acteurs pour l'utilisation et l'appropriation des pratiques et instruments organisationnels et de gestion injectés par ces organisations.

\section{Implantation de ChildFund et dynamiques d'organisation des communautés dans le département de Ziguinchor}

\subsection{Les étapes de la mise en place du réseau associatif de ChildFund à Ziguinchor}

ChildFund est une organisation internationale basée dans la ville de Richmond, aux États-Unis, et présente au Sénégal (à Dakar) depuis 1985. Ses interventions sont centrées autour du parrainage des enfants. C'est en 2002 que l'ONG a étendu son action dans le département de Ziguinchor. Son intervention a démarré dans la capitale départementale autour d'un programme d'appui psychosocial au bénéfice des familles victimes du conflit. Après une année de collaboration avec la mairie de Ziguinchor qui hébergeait ses programmes entre 2002 et 2003, l'ONG a créé l'association eluup añil, la maison de l'enfant en diola. Cette collaboration était une phase pilote, selon un ancien mobilisateur, qui a permis aux agents recrutés par l'ONG d'entrer en contact avec la communauté entendue comme bénéficiaire des programmes de ChildFund dans la ville de Ziguinchor.

Pour les agents de terrain de la fédération (relais, mobilisateurs communautaires, staff technique), la création d'eluup añil était une stratégie de l'ONG basée sur des services directs, c'est-à-dire des interventions qui parvenaient aux bénéficiaires regroupés 
dans l'association, sans passer par des intermédiaires. Dans sa démarche, ChildFund a très tôt mis en place un dispositif organisationnel qui mobilise divers outils administratifs. Dès les premières activités de l'association, plusieurs documents (fiches de présence, comptes rendus, rapports et justificatifs de dépenses, etc.) étaient produits ou mis à la disposition des agents de terrain. D'une part, ces documents permettaient à l'ONG d'assurer le suivi de ses interventions et la traçabilité des financements octroyés à l'association. D'autre part, leur production et leur utilisation régulière (une routinisation des pratiques des acteurs) étaient une forme de contrôle exercé par ChildFund sur l'association et le travail des agents de terrain.

Toutefois, c'est l'introduction du parrainage des enfants en 2005 qui a joué un rôle prépondérant dans la constitution du réseau associatif de l'ONG dans le département de Ziguinchor. Le parrainage consiste à trouver des volontaires (américains, hongkongais, français, etc.) qui acceptent de soutenir et d'appuyer des enfants issus de familles dites « démunies, exclues et vulnérables » à travers des cotisations mensuelles, une prise en charge scolaire et un soutien sanitaire (cotisation dans une mutuelle de santé). Il est piloté par le bureau international de ChildFund (instance de prise de décision) basé à Richmond. Les décisions et planifications qui y sont faites sont ensuite transmises au bureau national, à Dakar, avant d'arriver au niveau local où elles sont mises en application:

Il y'a un quota que le bureau international alloue pour le Sénégal. Ce quota est réparti entre les fédérations. Chaque fédération travaille avec les associations dans sa zone d'intervention. Le quota alloué à Ziguinchor est réparti entre les six associations. Chaque association veille à la bonne gestion de son quota : faire un suivi du mouvement des enfants parce que si un enfant parrainé quitte un village pour aller dans une zone où le projet n'intervient pas, il doit être retiré du programme et remplacé par un autre. (S.B responsable du parrainage)

Le bureau international conçoit les programmes, réceptionne les cotisations des parrains (vingt-cinq dollars US par mois), alloue les budgets au bureau national qui les distribue aux bureaux locaux constitués par les fédérations ${ }^{8}$, dont celle de Ziguinchor. Les budgets sont accordés proportionnellement au nombre d'enfants parrainés dans la zone d'intervention des différentes fédérations qui assurent leur exécution. De leur côté, les fédérations définissent les critères d'éligibilité au parrainage (enfants issus de familles DEV, c'est-à-dire sans emploi ou à revenu faible, familles déplacées, signer une convention de parrainage avec l'ONG, etc.) selon les contextes, et sélectionnent les enfants. Dans le suivi de ce parrainage, les agents de terrain étaient confrontés à plusieurs difficultés :

On avait souvent des difficultés à respecter le timing pour écrire les correspondances, c'est-à-dire les lettres que les enfants doivent envoyer à leurs parrains pour accuser réception d'un cadeau, répondre à une lettre, prendre des nouvelles, etc. Les parents étaient réticents et cela ne facilitait pas notre travail.

\footnotetext{
8 L'ONG compte cinq fédérations appelées local partners (LP) réparties à Dakar, Mbour, Thiès, Diourbel et Ziguinchor.
} 
Ils ne pouvaient pas croire qu'un parrain du Nord puisse envoyer deux dollars américains à son filleul au Sénégal. Ils pensaient que l'argent était détourné par les agents de terrain. Cela ne les motivait pas à réunir les enfants pour la rédaction des correspondances. (I.B ancien mobilisateur communautaire)

Ainsi, selon les agents de terrain, les parents estimaient que les montants reçus par leurs enfants étaient très petits ; ils ne trouvaient pas leur intérêt dans la relation de parrainage et le temps consacré à la rédaction d'une lettre paraissait inutile. Pour y remédier, les agents de terrain ont mis en place des cellules locales présentées comme des « instances de gestion du parrainage ». Avec ces cellules issues de l'association éluup añil dont elles étaient des démembrements, les agents n'avaient plus besoin de faire des visites à domicile ou de convoquer les enfants et leurs parents qui reportaient constamment les rencontres de rédaction des correspondances. Ils pouvaient passer désormais par ces cellules où se retrouvaient les parents pour ensuite convoquer leurs enfants. Aujourd'hui, cette fonction des cellules locales est assurée par les associations villageoises d'épargne et de crédit (AVEC) installées progressivement à partir de 2012.

En 2009, l'ONG a étendu ses interventions en milieu rural et périurbain suite à une revalorisation du quota de Ziguinchor qui est passé de 2000 (en 2005) à 5000 (en 2009) enfants à parrainer. Cette extension s'est accompagnée d'une réadaptation de la stratégie de l'ONG : les services directs sont remplacés par des services communautaires, c'est-à-dire des programmes (éducatifs, audiences foraines, campagnes de sensibilisation, renforcements de capacité, etc.) qui profitent à la fois aux parents et enfants parrainés et à ceux qui ne le sont pas. Elle a conduit à une redéfinition du concept de communauté qui renferme désormais un sens plus large : il renvoie aux parents et aux enfants (parrainés ou non) de la zone d'intervention de la fédération dimbaya. Cette nouvelle approche que les agents qualifient de « communautaire » s'inscrit dans une dynamique de gestion concertée (Méral, Castellanet, Lapeyre, 2008), mais surtout de légitimation et de rationalisation qui conduit l'ONG à mobiliser la communauté dans la mise en œuvre de ses activités. Elle a abouti à la création d'un réseau de six associations et de plus de deux cents AVEC. Ces associations sont regroupées dans la fédération dimbaya en 2009 en remplacement de l'association eluup añil qui n'était plus fonctionnelle depuis 2008. 


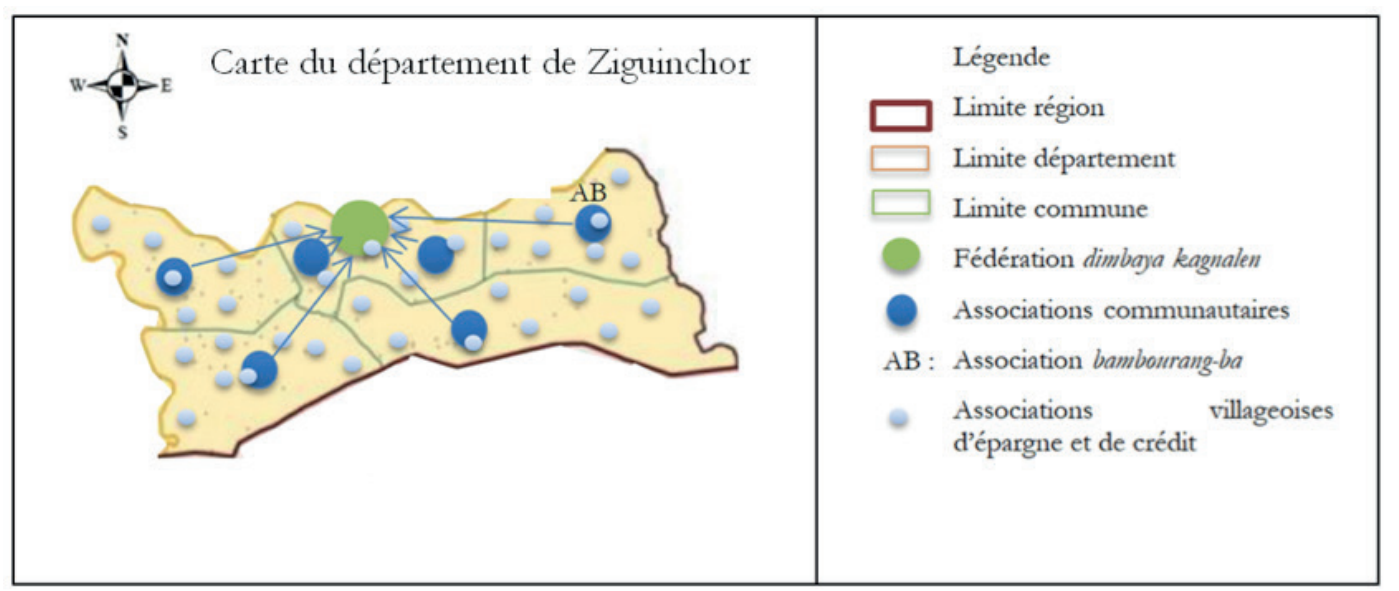

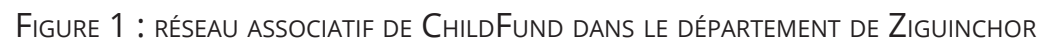
SOURCE : DONnÉES DE TERRAIN, JANVIER 2020

Cette carte situant géographiquement les structures associatives mises en place par ChildFund offre un aperçu approximatif sur la présence de l'ONG dans le département de Ziguinchor. Cette dernière a conçu et mis en place une nomenclature d'organisation qui permet de distinguer les niveaux départemental, communal et villageois. Dans le département, elle est représentée par la fédération dimbaya. Dans les communes, elle interagit avec les associations communautaires, dont bambourang-ba. Chaque association est représentée par les AVEC créées dans les quartiers et les villages d’intervention de la fédération. Ce réseau associatif est régi par des textes règlementaires dont le modèle et les bases sont définis par ChildFund.

\subsection{Ancrage et fonctionnement de l'association bambourang-ba}

Bambourang-ba est une association communautaire créée en 2009 et qui regroupe les habitants des villages de la commune rurale d'Adéane. Elle suit l'organisation formaliste de la fédération dimbaya avec une assemblée générale, un conseil d'administration et un bureau constitué d'un président, d'un secrétaire et d'un trésorier. Son assemblée générale est composée des (trois) représentants de chaque village. Les parents bénéficiaires du même village se réunissent pour choisir leurs représentants à l'issue d'une élection organisée à cet effet et appelée " assemblée sectorielle » :

Avant les renouvellements, nous tenons des rencontres avec les chefs de village et les autorités de la commune pour leur dire que ce bureau est venu à son échéance. Chaque village se réunit pour choisir ses représentants dans l'assemblée générale qui sera constituée. Ce sont ces élections organisées dans les villages qu'on appelle assemblée sectorielle [...]. (M.T mobilisateur communautaire)

Les premières assemblées sectorielles ont démarré avec la création de l'association. Leur organisation est encadrée par le staff de la fédération et le bureau sortant de l'association en présence des autorités locales (chef de village, maire, ICP) ou de leurs 
représentants. Elle obéit à des principes administratifs formalisés. Les assemblées sont tenues sur la base d'un calendrier triennal (qui n'est pas toujours respecté) inscrit sur l'agenda de l'association. Elles répondent à une exigence d'ancrage communautaire qui vise à accroître la légitimité des programmes et activités de l'ONG dans la commune d'Adéane. Elles offrent également un aperçu sur la gestion de l'association qui s'apparente à un modèle hybride partagé entre une gouvernance interne et une gouvernance externe.

La première est assurée par les acteurs locaux, élus lors de l'assemblée générale de l'association. Ces derniers se chargent, entre autres, de la mobilisation des communautés et la production des justificatifs de dépenses. Ils servent d'intermédiaires entre la fédération et les agents de terrain d'un côté, et les communautés de l'autre. Cette forme de gouvernance répond à une « logique de représentativité » (Chatelain-Ponroy, Eynaud, Sponem, 2014 : 1) qui facilite l'accès de l'ONG aux communautés. Quant à la seconde, elle reproduit le «schéma de planification [...] insufflée par les ONG ou les bailleurs de fonds (logique top-down)» (Méral, Castellanet, Lapeyre, 2008 : 8). Elle est pilotée de l'extérieur par ChildFund et la fédération dimbaya qui exercent un pouvoir de contrôle sur les activités mises en œuvre par l'association. Ce modèle de gouvernance est justifié par les agents de terrain qui estiment que les associations sont « immatures » et « incapables de se gérer toutes seules ». Il conduit à une hiérarchisation des relations entre les agents de terrain et les responsables de l'association.

Ces considérations ne sont pas toujours partagées par les responsables de l'association, dont certains mettent en avant leur expérience dans la gestion des associations villageoises. Lors de l'entretien que nous avons réalisé avec lui, l'ancien président rappelait régulièrement " ses trente ans d'expérience dans l'association des jeunes d’Adéane pour le développement ». Pour lui, cette expérience et la mobilisation de son réseau de relations personnelles ont permis « d'ancrer l'association dans la commune d'Adéane et d'assurer son bon fonctionnement pendant trois mandats ». De son côté, le président sortant ne manquait pas de mentionner son statut de conseiller à la mairie d'Adéane et les neuf ans passés à la tête du secrétariat avant d'occuper le poste de président de l'association. Il évoque toutefois la dépendance de celle-ci vis-à-vis de l'ONG et de la fédération. Il souligne qu'au « début, c'est la fédération qui venait pour dire ce qu'il faut faire en termes d'activités et de planification. Après, ils ont dit qu'il faut donner le pouvoir à l'association, il faut donner les compétences à l'association. Mais tout ce qu'on fait vient de la fédération ».

L'emploi des expressions « donner le pouvoir », « donner les compétences » par le président sortant traduit l'existence d'un pouvoir de décision et de planification monopolisé par l'ONG et la fédération dimbaya. Cette tendance est confirmée par le responsable du parrainage :

Il y a une planification qui se fait au niveau central par l'ONG et la fédération. C'est à partir de ces planifications que nous recevons les fonds qui permettent de réaliser les activités avec les associations. Donc, en tant qu'appui technique, 
nous devons apporter toute la technicité pour une bonne exécution des activités sur le terrain. (S.B responsable du parrainage)

Le staff technique conçoit un plan triennal duquel sont issus les plans d'opération annuelle des associations, dont ceux de bambourang-ba. Ils sont ensuite partagés avec le bureau et servent de programme annuel à l'association. Le budget de l'association est voté par l'assemblée générale de la fédération en présence du président. Il est accordé en fonction de ce programme. Son exécution se fait sous le contrôle et l'appui du staff technique et des agents de terrain de la fédération.

\section{3. « Coacher » l'appropriation des pratiques bureaucratiques}

Le modèle d'organisation et le système de contrôle de ChildFund mobilisent des outils administratifs et procéduraux variés. Comme le soulignent le président sortant et son secrétaire, « il faut écrire un procès-verbal après chaque réunion, produire une liste de présence et un rapport pour chaque activité et justifier chaque dépense réalisée par l'association » :

Après chaque activité, on doit déposer les justificatifs des dépenses et les différents documents. On vérifie d'abord avec le mobilisateur communautaire que tout a été dépensé dans les règles de l'art et les documents qui le justifient sont là ; on fait la vérification de tous ces documents avant de les envoyer au responsable financier de la fédération. (M.S président sortant de l'association)

Interrogés sur l'utilité de ces rituels bureaucratiques, les agents du staff technique de la fédération soutiennent qu'ils visent à assurer le bon déroulement et le suivi du parrainage. Toutefois, leur utilisation et leur appropriation par les communautés sont une condition pour l'intervention de l'ONG et s'inscrivent dans la durée. Elles conduisent à une routinisation des pratiques devenues normales du point de vue des membres et dirigeants de l'association. Il s'agit d'un processus continu qui a démarré avec la création de l'association et qui s'intègre peu à peu dans son fonctionnement par le travail des agents de terrain, notamment les mobilisateurs communautaires. En effet, outre la médiation qu'ils assurent entre l'association, la fédération, et l'ONG, ces agents jouent le rôle d'accompagnateurs et d'appui technique pour s'assurer du respect et de la mâ̂trise des standards organisationnels et administratifs de l'ONG :

Je suis chargé d'aider l'association dans les tâches administratives : suivi et orientation par rapport au bailleur et au partenaire local. Je l'accompagne dans la mise en œuvre des projets, des activités, dans la discussion avec d'autres partenaires. Ma mission est un coaching, un accompagnement pour produire des termes de référence, faire un état financier, écrire des rapports ; c'est d'aider l'association dans ses relations avec les élus locaux et les autres leaders; de les aider dans la rédaction de lettres. (M.T mobilisateur communautaire)

Ainsi, le mobilisateur communautaire assure à lui seul l'essentiel des tâches administratives de l'association. Il se substitue, de fait, au bureau élu dont la participation et l'implication paraissent essentiellement théoriques. D'ailleurs, selon un ancien agent 
de terrain, les mobilisateurs sont des jokers ${ }^{9}$ qui ont pour mission principale le coaching de l'association communautaire pour l'utilisation et l'appropriation des pratiques et rituels administratifs. Cette mission de « coaching » et « d'accompagnement » est inscrite dans le cahier des charges des agents de terrain, dont le recrutement semble tenir compte des expériences passées dans le domaine de l'administration et la maîtrise des procédures de gestion administrative.

Sur les quatre mobilisateurs interviewés, trois ont travaillé dans des services publics ou projets de développement en tant que relais communautaires, c'est-à-dire des agents chargés de faciliter les flux de communication et les échanges entre différents partenaires (Blundo, 2000 : 74). Parmi ces agents, deux ont suivi des études en administration et gestion de projets. Après son recrutement, chaque agent bénéficie d'une formation et d'un stage d'application sur les procédures de suivi et de gestion du parrainage avant d'être affecté à un poste. Ainsi, avant d'entamer sa fonction " d'agent de terrain », le mobilisateur accumule des connaissances sur les rouages administratifs qu'il transmet à son tour aux responsables de l'association lors des ateliers de formation dédiée, des réunions mensuelles, des rencontres d'échange et de partage. Il devient ce qu'on pourrait appeler un bureaucrate de haut niveau (O’Connor, 2017 : 70).

Ce mode d'intégration et d'intériorisation des procédures de gestion associative ressemble à un " idéal-type d'organisation » (Lavigne Delville, 2011 : 164) recherché par ChildFund et « tout projet dans les pays en développement » (Olivier de Sardan, 2009 : 26). Il reproduit le modèle d'une organisation dans laquelle bambourang-ba est le relai d'une chaîne administrative descendante (Dubet, 2016). Cependant, la cohérence qui s'affiche au travers de cette chaîne et qui semble tenir grâce au coaching des agents de terrain ne reflète pas toujours la vie quotidienne de l'association et de ses démembrements, notamment les AVEC. Dans le fonctionnement de ces dernières, les instruments et pratiques bureaucratiques (comité de gestion, système d'enregistrement et d'archivage des épargnes et crédits, règlementation/formalisation de la solidarité, etc.) ne sont pas toujours intégrés. Les membres adoptent une partie du « programme » et rejettent les pratiques qui ne corroborent pas les habitudes locales en matière d'organisation associative et de pratique de l'épargne.

Cette stratégie de «l'adoption sélective » (Olivier de Sardan, 1995 : 175) permet aux membres des AVEC de retenir une partie des pratiques qui leur conviennent subjectivement et qui fonctionnent pour eux. Ainsi, la mise en place d'une caisse de solidarité formalisée à l'endroit des parents bénéficiaires est confrontée aux limites de la " parenté ». Dans les considérations des communautés, la parenté inclut les grands-parents ainsi que les cousins (paternels et maternels) et leurs familles. Or, dans l'approche de l'ONG et de la fédération, la parenté se définit par le lien de sang et se limite au couple et aux enfants biologiques. Cet écart entre le système de valeur des acteurs locaux et des ins-

\footnotetext{
${ }^{9}$ Pour cet agent, le statut de joker se justifie parce que les mobilisateurs sont impliqués dans les différentes activités et interviennent dans tous les programmes de la fédération et de l'ONG. Pour lui, « il faut savoir tout faire, être un passe-partout, pour être mobilisateur communautaire de la fédération dimbaya ».
} 
truments théoriques de l'ONG mobilisés par les agents de terrain traduit les difficultés d'adaptation du modèle hiérarchisé de ChildFund en milieu rural ziguinchorois.

\section{Intermédiations et bureaucratisation dans le parrainage}

Le parrainage des enfants est l'une des principales activités de ChildFund et de dimbaya dans le département de Ziguinchor. Il repose sur l'établissement du lien parrain/parrainé qui peut prendre des formes variées (Blain et al., 2019). Le maintien de la relation de parrainage donne lieu à une série d'intermédiations assurées par l'ONG, la fédération et les agents de terrain. L'ONG et la fédération sont les garants de la convention qui lie les enfants à leurs parrains. Elles contrôlent les contenus des correspondances de manière échelonnée (de l'ONG aux agents de terrain ou inversement selon que la correspondance soit envoyée par un parrain ou un enfant), interrompent les relations qui ne respectent pas les termes du contrat de parrainage et exigent des parrains et des enfants (et leurs parents) un engagement formalisé, à l'image de la déclaration ci-dessous extraite de la deuxième page du formulaire $n^{\circ} 2^{10}$ de ChildFund et de dimbaya :

Je soussigné [...] déclare comprendre les activités auxquelles mon enfant va participer dans le Projet de parrainage. [...] J'autorise mon enfant à recevoir de la correspondance de son parrain (marraine) et à lui écrire en retour. J'accepte que toutes les correspondances en provenance ou à la destination du parrain (marraine) passeront par la Fédération/Bureau national et seront ouvertes et examinées par les agents de la Fédération/Bureau national. [...] et qu'en aucun cas l'adresse de mon parrain ne doit m'être transmise et que la communication directe avec mon parrain est interdite et est aussi motif de retrait du programme [...].

Cependant, le contrôle et les différentes intermédiations qui transparaissent dans cet extrait n'expriment pas une simple « manipulation professionnelle de personnes et d'informations » (Boissevain, 1974 : 148) ou une posture stratégique des agents pour capter les financements des projets de développement (Bierschenk, Chauveau, Olivier de Sardan, 2000). En effet, le lien de parrainage établit une relation d'interaction entre deux catégories d'acteurs qui n'ont pas nécessairement les mêmes représentations et les mêmes attentes. D'un côté, il y a le parrain « du Nord » qui exprime son aide et sa solidarité à son filleul ; il privilégie la « dimension humanitaire » du parrainage (Blain et al., 2019: 213). De l'autre, il y a l'enfant « du Sud» et sa famille qui formulent des attentes vis-à-vis du parrain supposé avoir une situation économique et financière plus stable. Ces attentes des enfants et de leurs familles découlent du mythe du « toubab riche ». Lors d'un entretien, une femme soutient que sa petite fille ne répond plus aux correspondances de son «toubab » qui n'envoie plus de cadeau depuis près d'un an. Pour elle, rompre la communication est un moyen d'exprimer sa déception sur le parrainage de son enfant.

\footnotetext{
${ }^{10}$ Le formulaire $\mathrm{n}^{\circ} 2$ est une fiche de renseignements utilisée lors du recensement des enfants dans le programme de parrainage. II est signé par les parents qui acceptent que leurs enfants soient inscrits dans le programme de parrainage de ChildFund et de la fédération dimbaya.
} 
Enfin, la série d'intermédiations auxquelles conduit le lien de parrainage donne lieu à un travail de bureaucratisation et de ritualisation des pratiques autour de l'encadrement des enfants pour la rédaction des correspondances telles que la welcome letter, la thank you letter, la bonding letter, la Child initiative letter (CIL) et des rapports de progrès annuels (RPA $)^{11}$. Comme l'a souligné un ancien mobilisateur, « le gros du travail du suivi du parrainage c'est la production de ces documents ». Ils se multiplient et se diversifient durant la relation de parrainage ; l'enjeu étant d'assurer le suivi et la pérennisation de cette relation. Les lettres sont écrites en présence de l'agent de terrain qui vérifie leur contenu et corrige les incorrections avec l'enfant. Dans le cas des RPA, les parrains hongkongais exigent l'utilisation d'un modèle de rapport écrit en chinois et français qu'ils ont conçu eux-mêmes sur la base des standards de l'ONG. Ces jeux d'acteurs témoignent de la complexité du lien de parrainage et de l'utilité des instruments et pratiques bureaucratiques, dont les significations varient selon les acteurs et les contextes.

\section{Conclusion}

Dans cet article, nous avons montré comment une association villageoise pouvait devenir un espace de bureaucratisation et d'appropriation des pratiques bureaucratiques à partir de l'exemple de l'association bambourang-ba. La création de cette dernière dans la commune d'Adéane s'est inscrite dans une dynamique globale d'organisation des bénéficiaires des programmes de l'ONG ChildFund qui a conduit à la mise en place d'un réseau de six associations communautaires dans le département de Ziguinchor. Son fonctionnement repose sur un ensemble d'instruments et d'outils de gestion administratifs insufflés par le haut. Pour l'ONG et la fédération dimbaya, l'enjeu de l'appropriation de ces instruments est d'exercer un contrôle sur l'association et d'assurer le suivi et le bon déroulement de leurs activités autour du parrainage des enfants.

Sous ce rapport, la bureaucratisation des pratiques associatives en milieu rural se présente comme une exigence pour ChildFund et dimbaya. Cette conditionnalité s'est traduite par la mise en place de structures locales (l'association bambourang-ba, les AVEC) «plus ou moins autonomes et responsabilisées sur certains secteurs d'activités » (Lavigne Delville, 2011 : 163), mais qui restent globalement dépendantes dans les faits. En outre, l'adhésion au modèle d'organisation bureaucratique ainsi mis en place et l'utilisation des instruments administratifs sont révélatrices d'une participation forcée des communautés qui sont obligées de suivre les canevas tracés par l’ONG et la fédération.

\footnotetext{
${ }^{11}$ La welcome letter marque le début de la relation de parrainage. Elle est écrite par l'enfant pour remercier le parrain de l'avoir accepté comme filleul. La thank you letter est une lettre de remerciement écrite par l'enfant après la réception d'un cadeau. La bonding letter est généralement produite tous les deux mois et permet de faire le suivi sur les relations actives et de réactiver celles qui ne le sont pas. Les CIL ou LS sont des lettres ordinaires qui peuvent être initiées par le parrain ou l'enfant pour prendre des nouvelles de l'autre. Enfin, les RPA sont des rapports annuels sur la situation de l'enfant : résultats scolaires, situation sanitaire, participation à des formations, etc. Le RPA est produit par l'agent de terrain sur la base d'une enquête auprès de l'enfant et ses parents.
} 
Cette bureaucratisation est toutefois confrontée aux dynamiques locales d'organisation qui se distinguent par leur caractère principalement informel. Le « coaching » et «l'accompagnement » des agents de terrain sont confrontés « aux principes d'adoption sélective et de détournement »(Olivier de Sardan, 1995 : 176) des pratiques bureaucratiques et du modèle d'organisation descendante privilégié par ChildFund et dimbaya. Cette stratégie se justifie du fait des écarts entre le corpus théorique des agents de terrain et les instruments de gestion insufflés par l'ONG et la fédération d'un côté, et les représentations et conceptions des communautés de l'autre. Elle conduit à une réorientation de certains programmes (tels que le projet AVEC) en fonction des attentes des communautés, mais aussi une réappréciation de l'utilité et de la signification des instruments bureaucratiques qui permettent d'encadrer et de maintenir le lien de parrainage. Enfin, elle pose la question des limites de la rationalité bureaucratique dans ses capacités à s'intégrer dans les pratiques des associations villageoises caractérisées par leur flexibilité (informelle) et l'oralité de leurs règles de fonctionnement.

\section{Bibliographie}

AwENEngo S. (2006), « À qui appartient la paix ? », Journal des anthropologues, $n^{\circ}$ 104-105. En ligne, consulté le $1^{\text {er }}$ mai 2019. URL : http://journals.openedition.org/jda/442.

BADIE B. (1992), L'État importé. L'occidentalisation de l'ordre politique, Paris, Fayard ( L'espace du politique »).

Bayart J.-F. (dir.) (1996), La greffe de l’État, Paris, Karthala (« Hommes et sociétés »).

Behrends A., Sung-Joon P., Rottenburg R. (2014), Travelling models in African conflict resolution: translating technologies of social ordering, Leiden, Brill (" Africa-Europe group for interdisciplinary studies $»)$.

Bierschenk T., Chauveau J.-P., Olivier de Sardan J.-P. (2000), Courtiers en développement. Les villages africains en quête de projets, Paris, Karthala.

Blain M.-J., Rodriguez del Barrio L., Caron R. et al. (2019), « Expériences de parrainage collectif de personnes réfugiées au Québec: perspectives de parrains et de personnes réfugiées de la Syrie », Lien social et Politiques, $n^{\circ} 83$. En ligne, consulté le 30 juin 2020. URL : https://id.erudit.org/iderudit/1066091ar.

Blundo G. (2000), «Élus locaux et courtiers en développement au Sénégal. Trajectoires politiques, modes de légitimation et stratégies d'alliance ", in T. Bierschenk, J.-P. Chauveau, J.-P. Olivier de Sardan (dir.), Courtiers en développement. Les villages africains en quête de projets, Paris, Karthala, p. 71-100.

Blundo G., Olivier de SARDAN J.-P. (dir.) (2007), État et corruption en Afrique: une anthropologie comparative des relations entre fonctionnaires et usagers (Bénin, Niger, Sénégal), Paris, Karthala (« Hommes et sociétés »).

Boissevain J. (1974), Friends of Friends, Networks, Manipulators and Coalitions, Oxford, Basil Blackwell. 
Chatelain-Ponroy S., Eynaud P., Sponem S. (2014), « La gouvernance associative : entre diversité et normalisation », in A. Burlaud (dir.), Comptabilité, Finance et Politique. De la pratique à la théorie : l'art de la conceptualisation, Paris, ECS, p. 219-226.

Chauveau J.-P. (1994), " Participation paysanne et populisme bureaucratique. Essai d'histoire et de sociologie de la culture du développement », in J.-P. JAСOB, P. LAVIGNE Delville (dir.), Les associations paysannes en Afrique : organisation et dynamiques, Paris, APAD-Karthala ( Hommes et Sociétés »), p. 25-60.

Combessie J.-C. (dir.) (2007), La méthode en sociologie, Paris, La Découverte.

DubEt F. (2016), « Les instruments et l'institution : le cas de l'école», Sociologie du travail, vol. 58, $n^{\circ}$ 4. En ligne, consulté le 12 juin 2020. URL : http://journals.openedition. org/sdt/807.

Dupuy C. (1990), « Les associations villageoises au Sénégal : fonctions économiques et modalités de financement », Revue Tiers Monde, vol. 31, n 122, p. 351-375.

ÉBĖRE C. (2015), « La géopolitique du conflit en Casamance : les relations entre le Sénégal et la Gambie », in Conflit et paix en Casamance. Dynamiques locales et transfrontalières, Goree Institute, p. 55-74.

Foucher V. (2007), " "Tradition africaine" et résolution des conflits. Un exemple sénégalais », Politix, vol. 4, n 80, p. 59-80.

Hiвоu B. (dir.) (2013), La bureaucratie néolibérale, Paris, La Découverte (« Recherches »).

Lascoumes P., Le Galès P. (dir.) (2005), Gouverner par les instruments, Paris, Presses de Sciences Po (« Académique »).

LAVIGne Delville P.(2011), «Du nouveau dansla “participation”?Populismebureaucratique, participation cachée et impératif délibératif », in E. JUL-LARSEN, P.J. LAURENT, P.Y. LE MEUR et al. (dir.), Une anthropologie entre pouvoirs et histoire : conversations autour de l'œuvre de Jean-Pierre Chauveau, Paris, Karthala et IRD (Hommes et Sociétés), p. 161188.

Manga M. L. (2012), La Casamance dans l'histoire contemporaine du Sénégal, Paris, L'Harmattan.

Méral P., Castellanet C., Lapeyre R. (dir.) (2008), La gestion concertée des ressources naturelles. L'épreuve du temps, Paris, Karthala.

NiANG A. (2000), « Les associations en milieu urbain dakarois : classification et capacités développantes ", Afrique et Développement, vol. 25, n²1/2, Dakar, CODESRIA, p. 99159.

O’Connor K. (2017), «Quelles sont les idées et les motivations des bureaucrates dans les sociétés contestées sur le plan religieux ?", Revue internationale des Sciences administratives, vol. 83, $\mathrm{n}^{\circ}$ 1, IISA, p. 69-88.

OlIVIER DE SARDAN J.-P. (1995), Anthropologie et développement. Essai en socioanthropologie du développement, Paris, Karthala (« Hommes et sociétés»). 
Olivier DE SARDAN J.-P. (2008), « À la recherche des normes pratiques de la gouvernance réelle en Afrique », Discussion Paper, $\mathrm{n}^{\circ}$ 5, AFID et Irish AID.

Olivier de SARDAN J.-P. (2009), « Gouvernance associative et gouvernance chefferiale dans les campagnes nigériennes », in J.-P. Olivier De SARDAN, M. T. Alou, Les pouvoirs locaux au Niger, Tome 1 : À la veille de la décentralisation, Paris, Karthala («Voix d’Afrique »), p. 15-36.

Olivier de Sardan J.-P., Diarra A., Moha M. (2017), « Travelling models and the challenge of pragmatic contexts and practical norms. The case of maternal health », Health Res Policy Sys. En ligne, consulté le 20 août 2020. URL : https://doi.org/10.1186/s12961017-0213-9.

Robin N. (2006), « Le déracinement des populations en Casamance. Un défi pour l’État de droit », Revue européenne des Migrations internationales, vol. 22, n 1, CNRS, p. 153-181.

TRINCAZ P.X. (1984), Colonisation et régionalisme, Ziguinchor en Casamance, Paris, ORSTOM («Travaux et documents », $n^{\circ} 172$ ).

WEBER M. (2016), Les concepts fondamentaux de sociologie, Paris, Gallimard.

WeLLER J.-M. (2018), Fabriquer des actes d'État. Une ethnographie du travail bureaucratique, Paris, Economica (« Études sociologiques »). 